\title{
The evaluation of nocturia in patients with lower urinary tract symptoms suggestive of benign prostatic hyperplasia and the analysis of the curative effect after medical or placebo therapy for nocturia: a randomized placebo-controlled study
}

\author{
Zhigang Xue ${ }^{1,2}$, Yunhua Lin ${ }^{1}$, Yongguang Jiang ${ }^{1 *}$, Nengbao Wei ${ }^{2}$ and Jinwen $\mathrm{Bi}^{2}$
}

\begin{abstract}
Background: To study nocturia in patients with lower urinary tract symptoms (LUTS) suggestive of benign prostatic hyperplasia (BPH) after medical or placebo treatment.

Methods: Patients with LUTS suggestive of BPH from several community clinics were included. Patients completed the International Prostate Symptom Score (I-PSS) questionnaire and a 3-day voiding diary. Urinalysis, prostatespecific antigen (PSA) measurement, and prostate ultrasonography were performed. Nocturnal polyuria (NP) was defined as a nocturnal urine fraction exceeding one third of the daily urine output in elderly men. A total of 148 outpatients were randomized to drug treatment (tamsulosin) or placebo treatment. After 8 weeks of treatment, they were re-evaluated using a 3-day voiding diary, PSA measurement, prostate volume (PV), I-PSS, etc.

Results: The average I-PSS score was 20.3, storage symptom score was 11.7, voiding symptom score was 8.6, quality of life (QoL) score was 3.7, PV was $40.4 \pm 19.4 \mathrm{ml}$, and nocturnal urine volume (NUV) was $845.7 \pm 339.0 \mathrm{ml}$. The mean frequency of nocturia was $2.3 \pm 1.1$ per day, and $94 \%$ of the patients had a nocturia frequency of more than two times per day. Of these patients, $76.5 \%$ had NP. A significant correlation was found between NUV and the amount of water intake at night and $4 \mathrm{~h}$ before sleep $(r=0.419, P=0.002 ; r=0.302, P=0.031)$. Eighty patients were randomized to drug treatment (tamsulosin) and 68 patients were randomized to placebo treatment. The I-PSS score was $16.8 \pm 4.9$ to $19.3 \pm 5.0(p=0.002)$, the storage symptom score was $10.3 \pm 3.4$ to $10.7 \pm 3.4(p=0.007)$, and the voiding symptom score was $7.5 \pm 2.4$ to $8.6 \pm 2.3(p=0.003)$. The frequency of daytime urination was $7.5 \pm 2.6$ to $8.1 \pm 2.6(p=0.002)$, maximum urine volume $(\mathrm{ml})$ was $372.8 \pm 103.3$ to $302.8 \pm 119.3(p=0.007)$, and morning urine volume $(\mathrm{ml})$ was $280.5 \pm 111.7$ to $259.5 \pm 100.7(p=0.003)$. However, the frequency of nocturia score was $2.8 \pm 0.7$ to $3.0 \pm 0.6(p=0.306)$ and the nocturnal urine volume $(\mathrm{ml})$ was $800.7 \pm 323.0$ to $845.7 \pm 303.5(p=0.056)$, which did not change significantly. There were significant differences between the NP and non-NP groups in the duration of LUTS, first voided urine volume, daytime urination frequency, and the amount of water intake at night and $4 \mathrm{~h}$ before sleep.

\footnotetext{
*Correspondence: jyg_doctor@sina.com

'Department of Urology, Beijing Anzhen Hospital, Capital Medical University,

Beijing 100029, China

Full list of author information is available at the end of the article
}

(c) The Author(s). 2018 Open Access This article is distributed under the terms of the Creative Commons Attribution 4.0 International License (http://creativecommons.org/licenses/by/4.0/), which permits unrestricted use, distribution, and reproduction in any medium, provided you give appropriate credit to the original author(s) and the source, provide a link to the Creative Commons license, and indicate if changes were made. The Creative Commons Public Domain Dedication waiver (http://creativecommons.org/publicdomain/zero/1.0/) applies to the data made available in this article, unless otherwise stated. 
(Continued from previous page)

Conclusions: Among the symptoms of LUTS, the improvement rates for nocturia were the lowest after medical treatment for BPH. The a-blockers did not improve nocturia, which was a common symptom accompanying LUTS suggestive of $\mathrm{BPH}$. Our results showed that the prevalence of NP was $76.5 \%$ and that NP was significantly related to the amount of water intake during the evening and before sleep.

Trial registration: ISRCTN registry, Trial registration number (TRN): ISRCTN85509614, Date of registration: 30/10/ 2018. This trial was registered retrospectively.

Keywords: Benign prostatic hyperplasia, Nocturia, Nocturnal polyuria, a-Adrenoceptor antagonists

\section{Background}

Nocturia, is perceived as one single symptoms of all lower urinary tract symptoms (LUTS) by most men, which is highly prevalent and the most bothersome component [1]. It has led to significant morbidity and, occasionally, mortality. Scholars have pointed out that, this bothersome symptom is related to cause arousal from sleep, and two or more voids per night are usually considered lead to a decreased health-related quality of life [2]. Nocturia could result from many others disease and conditions (including urological and non-urological diseases) [3]. However, the various causes are not differentiated excluded in men with LUTS/BPH, and none of the individual $\alpha_{1}$-adrenoceptor antagonists without subtype selectivity have obvious benefit. The medical therapy have not shown a significant reduction in frequency of nocturia so far. There are also not equal amounts of studies showing positive or negative results on nocturia [4]. For all of these reasons, nocturia is often multifactorial in etiology, and many medical conditions are associated with nocturia. Therefore, we aim to explore nocturia in patients with lower urinary tract symptoms suggestive of benign prostatic hyperplasia, and we analyzed the curative effect after medical therapy for nocturia.

\section{Methods \\ Patients}

From January 2015 to May 2016, 148 patients were admitted to Beijing Huairou Community Health Service Center, with a median age of 69.0 years, a mean \pm standard deviation (SD) age of $62.1 \pm 13.6$ years, LUTS/BPH duration of $5.0 \pm 3.1$ years, and absence of treatment using $\alpha$-blockers and/or $5 \alpha$-reductase inhibitors. The common inclusion criteria for all participants were age $\geq 50$ years and diagnosis of $\mathrm{BPH}$. All patients provided their written informed consent and the program was accepted by the hospital ethics committee. The exclusion criteria were prostate cancer, PSA $>10 \mathrm{ng} / \mathrm{ml}$, urinary tract infection, nervous system disease, urolithiasis, medical therapy that could affect the function of urination, prostatic surgery, or pelvic surgery.

\section{Methods}

Based on the literature [5], we estimated that 74 patients will need to be recruited in order to detect a clinically significant difference of $25 \%$ between the 2 groups (with a power of $80 \%$ ). Based on past clinical trial experience, we estimated that $20 \%$ of randomized participants will be lost in follow up, therefore 88 patients will need to be recruited, and 170 participants who will complete the item in our study.

The patients included in the study were randomly divided (1:1) into two groups (drug treatment and placebo treatment) by use of a computer generated random number table. But some patients of the two groups had lost in follow-up because of financial status, phone lost, language barriers, individual patient factors, etc.

At last all 148 patients with LUTS suggestive of $\mathrm{BPH}$ were divided into two random groups, which either received $\alpha$-adrenoceptor antagonist (tamsulosin $2 \mathrm{mg}$ qd po) or placebo for 8 weeks. IPSS, QoL index questionnaire, 3-day frequency volume charts (FVCs), PSA, urinalysis, PV, and uroflowmetry (Qmax) were assessed before and after medical therapy. Serum PSA and urinalysis were evaluated at Beijing Huairou Hospital. The specific instrument for the evaluation of nocturia was a $1000 \mathrm{ml}$ graduated glass. This study was approved by the ethics committee of Beijing Huairou Hospital. Each patient was informed of the study and signed an informed consent form and had the right to withdraw from the study at any time.

\section{Nocturia evaluation index}

Nocturia, specifically, was the number of voids recorded during the nighttime [3]. The first morning void was excluded from the count because it is not followed by sleep. Nocturnal urine volume describes the amount of urine excreted during the nighttime and includes the volume of the first morning void because this urine has been produced during the nighttime [3]. Nocturnal polyuria is an abnormally large urine volume produced during the nighttime. The ICS classifies nocturnal polyuria as the nocturnal urine volume divided by the 24-h urine volume (i.e., nocturnal polyuria index, NPi) [3]. The ICS defines nocturnal polyuria as NPi $>33 \%$ in the elderly [3]. 


\section{Statistical analysis}

SPSS 22.0 statistical software was used for data analysis. All variables were expressed as the mean $\pm S D$ values. The numerical data were compared using the unpaired $t$-test. An analysis of the difference in the number of patients with nocturia before and after treatment was performed using McNemar's test. A multivariate analysis was performed. A P-value of 0.05 or less was considered significant.

\section{Results}

The clinical index and results of 3-day frequency-volume charts from patients with LUTS/BPH (Table 1) The relationship between NUV, NPi, and evening drinking volume $4 h$ before bedtime drinking volume and other indices (Fig. 1 and Fig. 2)

The relationship between NUV and evening drinking volume $(r=0.419, p=0.002)$ and $4 \mathrm{~h}$ before bedtime drinking volume $(r=0.302, p=0.031)$ were related. NPi and maximum urination volume $(r=0.440, p=0.001)$ and morning urine volume $(r=0.445, p=0.001)$ were related, but there was no correlation between NPi and prostate volume, age, LUTS duration, or I-PSS score.

Table 1 Clinical indices and nocturia-related parameters in patients with $\mathrm{BPH}$

\begin{tabular}{ll}
\hline Variable & $\begin{array}{l}\text { Median } \\
\text { (mean } \pm \text { SD) }\end{array}$ \\
\hline Number of patients & 148 \\
Age (years) & $69.5 \pm 5.6$ \\
LUTS/BPH duration (years) & $6.3 \pm 3.1$ \\
I-PSS & $20.3 \pm 5.0$ \\
Storage symptom score & $11.7 \pm 3.4$ \\
Voiding symptom score & $8.6 \pm 2.3$ \\
QoL score & $3.7 \pm 1.0$ \\
Nocturia score & $3.0 \pm 1.0$ \\
Serum PSA (ng/ml) & $2.0 \pm 1.7$ \\
Prostate volume (ml) & $40.4 \pm 19.4$ \\
NPi & $0.43 \pm 0.12$ \\
24-h drinking volume (ml) & $1565.4 \pm 624.8$ \\
4-h before bedtime drinking volume (ml) & $123.9 \pm 109.9$ \\
Frequency of nocturia & $3.3 \pm 1.1$ \\
Frequency of daytime urination & $8.1 \pm 2.6$ \\
Maximum urination volume (ml) & $352.8 \pm 119.3$ \\
Morning urine volume (ml) & $260.5 \pm 109.7$ \\
24-h total urine volume (ml) & $1996.5 \pm 637.5$ \\
Nocturnal urine volume (ml) & $845.7 \pm 339.0$ \\
\hline L & \\
\hline
\end{tabular}

LUTS lower urinary tract symptoms, $B P H$ benign prostatic hyperplasia, $P S A$ prostate-specific antigen, I-PSS, international prostate symptom score, NPi nocturnal polyuria index, QoL quality of life
The comparison of evaluation indices between drug treatment and placebo treatment groups for patients with $\mathrm{BPH}$ (Table 2)

Eighty patients were randomized to the drug treatment (tamsulosin) and 68 patients were randomized to the placebo treatment. The I-PSS score, storage symptom score, voiding symptom score, quality of life score, frequency of daytime urination, maximum urine volume, and morning urine volume were statistically significant between both groups. However, the frequency of nocturia score and nocturnal urine volume did not change significantly.

\section{Data statistics between the NP group and the non-NP group (Table 3)}

In the indices of age, 24-h drinking volume, bladder function, PV, and PSA level, there were no significant differences between the groups. While in LUTS duration, nocturnal urine volume, morning urine volume, and daytime voiding frequency, the differences between the two groups were statistically significant.

\section{Discussion}

Nocturia, one of the most bothersome symptoms of LUTS, has been the focus of a high volume of rapidly evolving research. The purpose of this study was to describe the relevant recent research in the field of nocturia in China, with particular emphasis on its evaluation and management. Nocturia is a complicated clinical entity that is often multifactorial in etiology. Experts of the International Continence Society (ICS) define nocturia as the general complaint when an individual (independent of age, gender, cause(s) and associated bother) must wake up at night one or more times to void $[1,2]$.

Epidemiological studies showed that the prevalence of nocturia in the <30-year-old population was approximately $3 \%$, in the 60 - to 69 -year-old population was $30 \%$, and in the $>70$-year-old population was $40 \%$ [6]. The survey regarding $>60$-year-old men in the United States demonstrated that up to $65.2 \%$ of old men would get up at night to urinate, of whom $25 \%$ of elderly males woke up at night to urinate $\geq 2$ times [7]. A Chinese questionnaire study showed that in patients living in the national scope, the storage symptoms of I-PSS were the most troubling symptoms of $\mathrm{BPH}$ in patients, in which nocturia was the most affecting [8]. Schatzl reported that more than $60 \%$ of old people thought nocturia would negatively affect their quality of life [6]. Recently, a study showed that the mortality rate of the elderly with nocturia was significantly higher (more than 3 times) than that of the elderly without nocturia (less than 3 times) [9].

Nocturia has been identified as the leading cause for sleep disturbance and sleep fragmentation; it causes daytime fatigue, impacts daily activities, and deteriorates psychomotor 


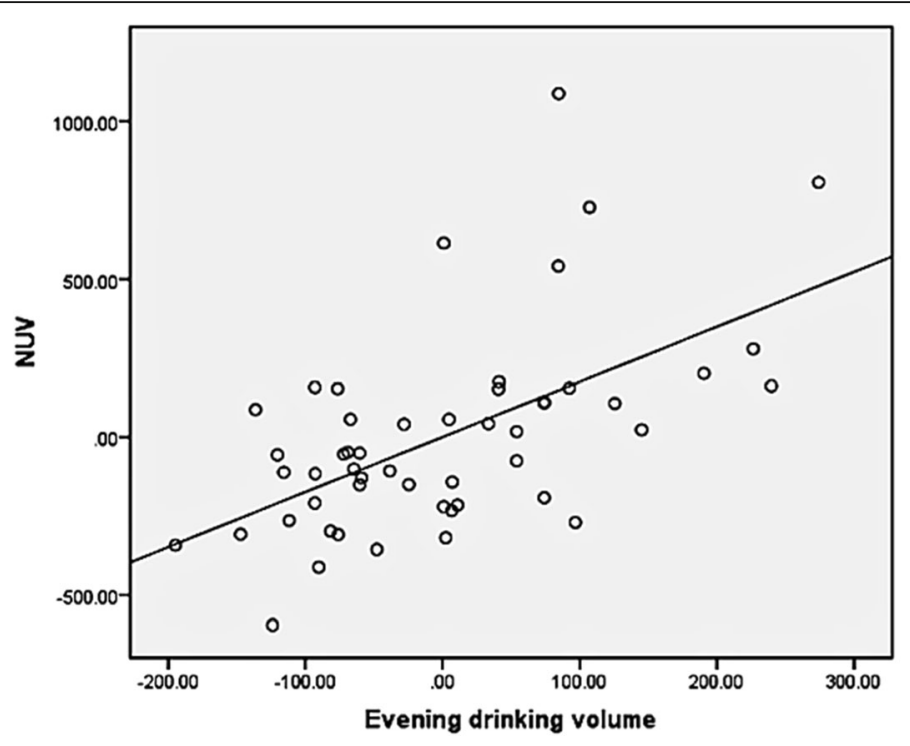

$r=0.419$

Fig. 1 The relationship between NUV and Evening drinking volume. 1) X axis caption: "Evening drinking volume (ml)". 2) Y axis caption: "NUV (ml)"

performance, cognitive function, and mood [10-12]. Nocturia can also cause depression and immunosuppression, and it increases the vulnerability for cardiovascular diseases and the development of diabetes mellitus [11, 13-15].

In these analyses, LUTS/BPH patients with nocturia had some urinary system diseases, such as lower urinary tract obstruction and overactive bladder, as well as cardiovascular diseases, diabetes, diabetes insipidus, and other awakening factors of urination (such as anxiety and sleep disorders). In this study, patients had hypertension, coronary heart disease, diabetes, and other diseases. Thus, nocturia may be caused by the combined effects of a variety of conditions. Kupelian et al. demonstrated that while increased odds of the metabolic syndrome were observed with mild to moderate degrees of nocturia [16]. In this way, the etiology of nocturia, especially, nocturnal polyuria can be further analyzed by evaluating LUTS patients with metabolic syndrome.

$\mathrm{BPH}$ is a common disease in elderly men, not only with voiding symptoms such as dysuria but also with storage symptoms such as urinary frequency, urgency, and nocturia. Clinical studies showed that the application of $\alpha$-blockers and $5 \alpha$-reductase inhibitors could effectively relieve lower urinary tract symptoms caused

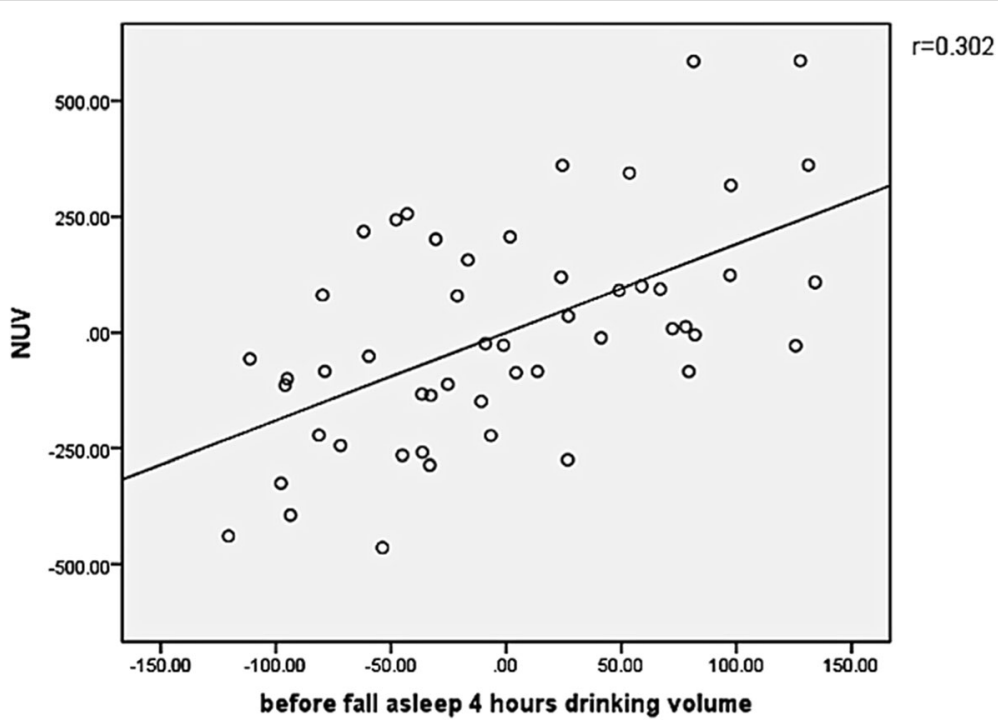

Fig. 2 The relationship between NUV and before fall asleep $4 \mathrm{~h}$ drinking volume. 1) X axis caption: "4-h before bedtime drinking volume (ml)". 2) Y axis caption: "NUV (ml)" 
Table 2 Comparison of evaluation indices after treatment (drug or placebo) in patients with BPH and nocturia

\begin{tabular}{|c|c|c|c|}
\hline Variable & Drug reatment & Placebo Treatment & $P$ value \\
\hline Number of patients & 80 & 68 & \\
\hline Age (years) & $69.5 \pm 5.6$ & $68.5 \pm 5.3$ & 0.450 \\
\hline I-PSS & $16.8 \pm 4.9$ & $19.3 \pm 5.0$ & 0.002 \\
\hline Storage symptom score & $10.3 \pm 3.4$ & $10.7 \pm 3.4$ & 0.007 \\
\hline Voiding symptom score & $6.5 \pm 2.4$ & $8.6 \pm 2.3$ & 0.003 \\
\hline QoL score & $3.1 \pm 0.6$ & $3.8 \pm 1.0$ & 0.005 \\
\hline Serum PSA (ng/ml) & $2.2 \pm 1.7$ & $2.1 \pm 1.6$ & 0.355 \\
\hline Prostate volume (ml) & $39.4 \pm 19.4$ & $41.4 \pm 20.1$ & 0.235 \\
\hline Qmax & $14.0 \pm 2.4$ & $16.5 \pm 2.6$ & 0.043 \\
\hline 24-h drinking volume (ml) & $1465.4 \pm 624.8$ & $1505.4 \pm 610.8$ & 0.356 \\
\hline 4-h before bedtime drinking volume ( $\mathrm{ml}$ ) & $115.8 \pm 100.9$ & $106.6 \pm 97.9$ & 0.452 \\
\hline Frequency of nocturia & $2.8 \pm 0.7$ & $3.0 \pm 0.6$ & 0.306 \\
\hline Frequency of daytime urination & $7.5 \pm 2.6$ & $8.1 \pm 2.6$ & 0.002 \\
\hline Maximum urination volume (ml) & $372.8 \pm 103.3$ & $302.8 \pm 119.3$ & 0.007 \\
\hline Morning urine volume (ml) & $280.5 \pm 111.7$ & $259.5 \pm 100.7$ & 0.003 \\
\hline 24-h total urine volume (ml) & $1965.5 \pm 623.5$ & $1896.5 \pm 637.5$ & 0.231 \\
\hline Nocturnal urine volume (ml) & $800.7 \pm 323.0$ & $845.7 \pm 303.5$ & 0.056 \\
\hline
\end{tabular}

LUTS lower urinary symptom, PSA prostate-specific antigen

by $\mathrm{BPH}$, but the efficacy in improving nocturia was not obvious $[17,18]$.

Tacklind thought that doxazosin and terazosin were significantly more effective than finasteride in improving nocturia, while no difference was found with tamsulosin [19]. This lack of specificity precluded prostatic surgery, such as transurethral resection of the prostate (TURP), from being the first-line therapy for nocturia. The symptoms of nocturia could not be improved in most patients. Nonetheless, various groups had reported decreases in nocturia after surgery in specific patient populations. In another study, Wada et al. evaluated the effect of TURP on nocturia in patients with LUTS/BPH. Nocturnal voids reduced from 3.0 to 1.9 per night after

Table 3 Comparison of evaluation indices in NP and non-NP patients

\begin{tabular}{|c|c|c|c|}
\hline \multirow[t]{2}{*}{ Variable } & \multicolumn{2}{|c|}{ Median (mean \pm SD) } & \multirow[b]{2}{*}{$P$ value } \\
\hline & $\mathrm{NPi} \geq 0.33$ & $\mathrm{NPi}<0.33$ & \\
\hline Number of patients & 113 & 35 & \\
\hline Percentage (\%) & 76.50 & 23.50 & \\
\hline Age (years) & $69.6 \pm 6.0$ & $69.1 \pm 4.0$ & 0.746 \\
\hline LUTS duration (years) & $5.8 \pm 2.7$ & $7.9 \pm 4.0$ & 0.043 \\
\hline 24-h drinking volume (ml) & $1931.9 \pm 680.6$ & $2206.5 \pm 429.1$ & 0.195 \\
\hline 4-h before bedtime drinking volume $(\mathrm{ml})$ & $140.8 \pm 135.8$ & $132.9 \pm 102.4$ & 0.029 \\
\hline Nocturnal urine volume (ml) & $922.4 \pm 342.6$ & $596.5 \pm 168.2$ & 0.003 \\
\hline Morning urine volume (ml) & $278.3 \pm 116.5$ & $202.5 \pm 55.0$ & 0.004 \\
\hline Frequency of daytime urination & $7.4 \pm 2.2$ & $10.6 \pm 2.4$ & 0.005 \\
\hline Bladder capacity index & $0.8 \pm 0.6$ & $0.8 \pm 0.6$ & 0.963 \\
\hline Maximum urination volume (ml) & $369.1 \pm 126.0$ & $299.4 \pm 75.7$ & 0.076 \\
\hline Prostate volume (ml) & $41.9 \pm 25.3$ & $39.5 \pm 24.8$ & 0.198 \\
\hline Peak flow rate (ml/s) & $13.2 \pm 10.0$ & $13.9 \pm 9.9$ & 0.12 \\
\hline Residual volume (ml) & $21.8 \pm 20.0$ & $20.6 \pm 18.7$ & 0.087 \\
\hline Serum PSA (ng/ml) & $4.7 \pm 4.1$ & $4.5 \pm 4.0$ & 0.102 \\
\hline
\end{tabular}


surgical treatment, which showed significant difference [5]. Yoshimura et al. reported that 505 cases of patients with BPH after drug treatment of tamsulosin and TURP surgery decreased the frequency of nocturia in patients by 17.9 and $32.2 \%$, respectively, but the efficacy in improving nocturia was not obvious [20]. Additional studies were warranted to validate and substantiate these findings. Though such treatments were unlikely to benefit most patients with nocturia, they could be tried in select cases.

In our study, 80 patients were randomized to drug treatment (tamsulosin) and 68 patients were randomized to placebo treatment. The scores and the data (include I-PSS score, storage symptom score, voiding symptom score, quality of life score, frequency of daytime urination, maximum urine volume, and morning urine volume) were all statistically significant. However, the frequency of nocturia score and the nocturnal urine volume did not change significantly. The traditional tool for evaluating nocturia was the I-PSS in nocturia subtype score, which was not described in detail [21]. Whatsmore, the important and detailed information of nocturia may not be fully captured by the I-PSS questionnaire alone. Michel et al. thought that the I-PSS (QoL and Nocturia score) can't show how nocturia decreases sleep quality [22]. Therefore, a thorough evaluation of any treatment of nocturia requires using a method for measuring the treatment impact on sleep quality and QoL [18]. The international consultation on incontinence questionnaire (ICIQ) N-QoL is developed by Abraham et al., and it is a validated questionnaire that can measure the deterioration of health-related quality of life (HRQoL) due to nocturia in general [23]. In our study, we also found it was just a basic assessment of the patient's own quality of life use I-PSS (QoL) score. On the one hand we worried about patients' coordination and understanding, on the other hand we ignored the importance of nocturia to quality of life. The (ICIQ) N-QoL et al. should be used to evaluate nocturia on quality of life in further study.

We used a 3-day urinary frequency voiding diary, including a 24-h urination, nocturnal urine volume, urine volume, total sleeping time, and amount of water intake for a detailed assessment of patients with nocturia to understand the correlation between nocturia and other factors. Therefore, the data that was obtained was in detail.

Koseoglu et al. reported 58 cases of patients with BPH, of whom $95 \%$ had NP. The frequency of nocturia was $2.73 \pm 1.44$ times/day, which confirmed that patients with BPH and NP had a relationship between LUTS duration and 3-h drinking of water before falling asleep [24]. In this study, patients with BPH were diagnosed as $\mathrm{BPH} / \mathrm{LUTS}$, which led to the decrease of a certain degree of bladder function, and all patients received treatment (drug treatment such as tamsulosin or placebo treatment). To some extent, medications could alleviate lower urinary tract symptoms in patients with $\mathrm{BPH}$, including storage symptoms such as urinary frequency and urgency. However, according to the analysis on 3 -day voiding diary results, NP was reported in $76.5 \%$ of patients with nocturia, which is lower than that reported for $95 \%$ of the patients with NP type nocturia. However, the proportion is still higher than other types of nocturia. Our results showed that there was no association between nocturnal urine volume and LUTS duration, PV, serum PSA, or I-PSS. However, there was a significant correlation between the amount of drinking water at night $(r=0.419, p=0.002)$ and before falling asleep 4-h drinking water $(r=0.302, P=0.031)$. Hence, we thought that the improvement of $\alpha$-blockers treatment for nocturia was poor.

In this study, between the NP group and the non-NP group, there was a significant difference in LUTS duration, maximum urine volume, morning urine volume, nocturnal urine volume, daytime urination, and 4-h before falling asleep drinking water volume. The frequency of daytime micturition and LUTS duration were the indices between the NP group and the non-NP group. A long disease history and more frequent daytime urination were considered the non-NP type, and $\alpha$-blocker drug treatment could improve this type of nocturia. Nocturia could also be alleviated by treating the other causes, such as increased water drinking before going to bed.

This study had some limitations, including a small sample size and the assessment with regard to short-term use of medication. Future studies of patients with LUTS/BPH and nocturia should, therefore, also include nocturia-specific instruments to correctly classify the underlying pathologies for nighttime voiding, and hence, provide a rationale for the treatment of all LUTS/BPH causes.

\section{Conclusions}

In conclusion, patients with lower urinary tract symptoms improved, to some extent, after receiving medication. However, the nocturia was not obviously improved and the incidence was still high. The patients could not achieve satisfactory results, and NP was highly prevalent among patients. The correlation between the frequency of nocturia and nighttime water intake and drinking water before going to bed were significant. We could control water intake to ease nocturnal urination.

\section{Abbreviations}

BPH: Benign prostatic hyperplasia; I-PSS: International Prostate Symptom Score; NP: Nocturnal polyuria; Npi: Nocturnal polyuria index

\section{Acknowledgements \\ Not applicable.}

Funding

Not applicable. 


\section{Availability of data and materials}

All data generated or analysed during this study are included in this published article.

\section{Authors' contributions}

ZGX, YHL, NBW and JWB obtained the data. ZGX and YGJ participated in designing of the study and performed the statistical analysis, and wrote the draft in the manuscript. All authors read and approved the final manuscript

\section{Ethics approval and consent to participate}

This study was approved by the ethics committee of Beijing Huairou Hospital. Each patient was informed of the study and signed an informed consent form and had the right to withdraw from the study at any time.

\section{Consent for publication}

Not applicable.

\section{Competing interests}

All authors' declare that they have no competing interests.

\section{Publisher's Note}

Springer Nature remains neutral with regard to jurisdictional claims in published maps and institutional affiliations.

\section{Author details}

'Department of Urology, Beijing Anzhen Hospital, Capital Medical University, Beijing 100029, China. ${ }^{2}$ Department of Urology, Beijing Huairou Hospital, Beijing 101400, China.

Received: 27 August 2018 Accepted: 26 November 2018

Published online: 13 December 2018

\section{References}

1. Austin PF, Bauer SB, Bower W, et al. The standardization of terminology of lower urinary tract function in children and adolescents: update report from the standardization committee of the international Children's continence society. Neurourol Urodyn. 2016;35(4):471-81.

2. Bosch JL, Weiss JP. The prevalence and causes of nocturia. J Urol. 2010; 184(2):440-6.

3. Van Kerrebroeck P, Abrams $P$, Chaikin D, et al. The standardisation of terminology in nocturia: report from the standardisation sub-Committee of the International Continence Society. Neurourol Urodyn. 2002;21(2): 179-83.

4. Asplund R. Nocturia: consequences for sleep and daytime activities and associated risks. Eur Urol Suppl. 2005;3(6):24-32.

5. Wada N, Numata A, Hou K, et al. Nocturia and sleep quality after transurethral resection of the prostate. Int J Urol. 2014;21(1):81-5.

6. Schatzl G, Temml C, Schmidbauer J, et al. Cross-sectional study of nocturia in both sexes: analysis of a voluntary health screening project. Urology. 2000:56(1):71-5.

7. Matthiesen TB, Ritting S, Norgaard JP, et al. Nocturnal polyuria and natriuresis in male patients with nocturia and lower urinary tract symptons. J Urol. 1996;156(4):1292-9.

8. Wang W, Chen S, Liu YX, et al. Understanding Chinese patient attitude toward BPH and BPH treatment: results from PHELP 2006 study. Urology. 2009;74(4):S48-8.

9. Asplund $\mathrm{R}$, Aberg $\mathrm{H}$. Health of the elderly with regard to sleep and nocturnal micturition. Scand J Prim Health Care. 1992;10(2):98-104.

10. Stanley N. The physiology of sleep and the impact of ageing. Eur Urol Suppl. 2005;3(6):17-23.

11. Abrams P. Nocturia: the effect on sleep and related health consequences. Eur Urol Suppl. 2005;3(6):1-7.

12. Torimoto K, Hirayama A, Matsushita C, et al. Evaluation of sleep quantity and quality in older adults with nocturia using portable electroencephalogram acquisition device. J Urol. 2013;189(4):e557-8,

13. Akerstedt T, Nilsson PM. Sleep as restitution: an introduction. J Intern Med. 2003;254(1):6-12.

14. Asplund R. Mortality in the elderly in relation to nocturnal micturition. BJU Int. 1999;84(3):297-301.

15. Bonnet MH, Arand DL. Clinical effects of sleep fragmentation versus sleep deprivation. Sleep Med Rev. 2003;7(4):297-310.
16. Kupelian V, McVary KT, Kaplan SA, et al. Association of lower urinary tract symptoms and the metabolic syndrome: results from the Boston area community health survey. J Urol. 2013;189(1 Suppl):S107-14.

17. Johnson TM, Burrows PK, Kusek JW, et al. Medical therapy of prostatic symptoms research group. The effect of doxazosin, finasteride and combination therapy on nocturia in men with benign prostatic hyperplasia. J Urol. 2007:178(5):2045-50.

18. Johnson TM, Williford WO, Kutner MH. Changes in nocturia from medical treatment of benign prostatic hyperplasia: secondary analysis of the Department of Veterans Affairs Cooperative Study Trial. J Urol. 2003;170(1):145-8.

19. Tacklind J, Fink HA, Macdonald R, et al. Finasteride for benign prostatic hyperplasia. Cochrane Database Syst Rev. 2010. https://doi.org/10.1002/ 14651858.CD006015.pub3.

20. Yoshimura K, Ohara H, Ichioka K, et al. Nocturia and benign prostatic hyperplasia. Urology. 2003;61(4):786-90.

21. Barry MJ, Fowler FJ, O'Leary MP, et al. The American urological association symptom index for benign prostatic hyperplasia. The Measurement Committee of the American Urological Association. J Urol. 1992;148(5):1549-57.

22. Michel MC, Chapple CR. Comparison of the cardiovascular effects of tamsulosin oral controlled absorption system (OCAS) and alfuzosin prolonged release (XL). Eur Urol. 2006;49(3):501-8.

23. Abraham L, Hareendran A, Mills IW, et al. Development and validation of a quality-of-life measure for men with nocturia. Urology. 2004;63(3):481-6.

24. Koseoglu H, Aslan G, Ozdemir l, et al. Noctural polyuria in patients with lower urinary tract symptoms and response to alpha-blocker therapy. Urology. 2006;67(6):1188-92.

\section{Ready to submit your research? Choose BMC and benefit from:}

- fast, convenient online submission

- thorough peer review by experienced researchers in your field

- rapid publication on acceptance

- support for research data, including large and complex data types

- gold Open Access which fosters wider collaboration and increased citations

- maximum visibility for your research: over $100 \mathrm{M}$ website views per year

At BMC, research is always in progress.

Learn more biomedcentral.com/submissions 